PROCEEDINGS OF THE

AMERICAN MATHEMATICAL SOCIETY

Volume 136, Number 2, February 2008, Pages 519-528

S 0002-9939(07)09036-3

Article electronically published on October 24, 2007

\title{
HYPERCYCLIC AND TOPOLOGICALLY MIXING COSINE FUNCTIONS ON BANACH SPACES
}

\author{
ANTONIO BONILLA AND PEDRO J. MIANA \\ (Communicated by N. Tomczak-Jaegermann)
}

\begin{abstract}
Our first aim in this paper is to give sufficient conditions for the hypercyclicity and topological mixing of a strongly continuous cosine function. We apply these results to study the cosine function associated to translation groups. We also prove that every separable infinite dimensional complex Banach space admits a topologically mixing uniformly continuous cosine family.
\end{abstract}

\section{INTRODUCTION}

A bounded linear operator $T \in \mathcal{B}(X)$ on a separable complex Banach space $X$ is said to be hypercyclic if there exists an $x \in X$ such that $\left\{T^{n} x\right\}_{n \in \mathbb{N}}$ is dense in $X$.

In 1969, Rolewicz 12 gave the first example of a hypercyclic operator on a Banach space. He showed that if $B$ is the backward shift on $l^{2}(\mathbb{N})$, then $\lambda B$ is hypercyclic if and only if $|\lambda|>1$. He also wondered if for every separable infinite dimensional Banach space there exists a hypercyclic operator. This question was independently answered in the affirmative by Ansari [1] and Bernal-González [5]. Bonet and Peris [7] generalized the result for Fréchet spaces.

It is well known that $T \in \mathcal{B}(X)$ is hypercyclic if and only if for any pair of nonvoid open sets $U, V \subset X$ there exists some positive integer $n_{0}$ such that

$$
T^{n_{0}} U \cap V \neq \emptyset \text {. }
$$

It is said that an operator is topologically mixing if the condition $(*)$ holds for every $n$ large enough.

A one-parameter family $\{T(t)\}_{t \geq 0} \subset \mathcal{B}(X)$ of bounded linear operators is a oneparameter semigroup of operators in $\mathcal{B}(X)$ if it verifies the following two conditions:

(i) $T(0)=I$,

(ii) $T(t) T(s)=T(t+s)$ for all $t, s \geq 0$.

Received by the editors July 17, 2006.

2000 Mathematics Subject Classification. Primary 47D09, 47A16.

Key words and phrases. Hypercyclic operators, topologically mixing operators, cosine functions, translation groups.

The first author is supported by MEC and FEDER MTM2005-07347 and MEC (Accion special) MTM2006-26627-E.

The second author is supported by Project MTM2004-03036, DGI-FEDER, of the MCYT, Spain, and Project E-64, D. G. Aragón, Spain. 
The infinitesimal generator $A$ of a semigroup $\{T(t)\}_{t \geq 0}$ can be calculated by

$$
A x:=\lim _{t \rightarrow 0^{+}} \frac{T(t) x-x}{t}
$$

for $x \in X$ where this limit exists. In the case where $A$ and $-A$ generate semigroups, it is said $A$ generates a group.

We say that a semigroup is strongly continuous or $C_{0}$-semigroup provided that $\lim _{t \rightarrow s} T(t) x=T(s) x$ for all $s \geq 0$ and $x \in X$. The semigroup is uniformly continuous if this limit holds uniformly on the unit ball of $X$. If $\{T(t)\}_{t \geq 0}$ is a strongly continuous semigroup, then the infinitesimal generator is closed and densely defined. In the uniformly continuous case, the infinitesimal generator is everywhere defined and bounded. See definitions and properties, for example, in [2].

A semigroup $\{T(t)\}_{t>0}$ is hypercyclic if there exists $x \in X$ such that $\{T(t) x\}_{t>0}$ is dense in $X$. We say that a semigroup $\{T(t)\}_{t \geq 0}$ is topologically mixing if for every pair of nonvoid sets $U, V \subset X$ there exists $t_{0}$ such that

$$
T(t) U \cap V \neq \emptyset \text { for all } t \text { such that } t \geq t_{0} .
$$

In [4] (3]) it is proved that every separable infinite dimensional complex Banach space admits a hypercyclic (topologically mixing) uniformly continuous semigroup. and

A family of operators $C(t): \mathbb{R} \rightarrow \mathcal{B}(X)$ is called a cosine function if $C(0)=I$

$$
2 C(t) C(s)=C(t+s)+C(t-s) \text { for all } s, t \in \mathbb{R} .
$$

To start, we assume that the family is strongly continuous, i.e., $t \rightarrow C(t) x$, for $t \in \mathbb{R}$, is a continuous $X$-valued function for each $x \in X$. The infinitesimal generator $A$ of $C(t)$ is defined by

$$
A x=\lim _{t \rightarrow 0} \frac{2}{t^{2}}(C(t)-I) x, \quad x \in D(A),
$$

where $D(A)$ consists of those vectors $x \in X$ such that the above limit exists. Then $A$ is a closed, densely defined operator. It is known that there exist constants $M>0$ and $w \geq 0$ such that $\|C(t)\| \leq M e^{w|t|}$ for all $t \in \mathbb{R}$. Moreover, if $z \in \mathbb{C}$ satisfies $\operatorname{Re} z>w$, then $z^{2} \in \rho(A)$ and so $\sigma(A)$ is contained in a parabolic domain in $\mathbb{C}$ which is open to the left [2, Proposition 3.14.18].

An operator $A$ generates a uniformly continuous cosine function $C(t)$; that is, $\lim _{t \rightarrow 0}\|C(t)-I\|=0$ if and only if $A$ is bounded. In this case the cosine function admits the following representation:

$$
C(t)=\sum_{n=0}^{\infty} \frac{t^{2 n}}{2 n !} A^{n} .
$$

Moreover, if $B$ is the generator of a $C_{0}$-group $T(t)$ on $X$, then $A:=B^{2}$ generates a cosine function $C(t)$ on $X$ given by

$$
C(t):=\frac{1}{2}(T(t)+T(-t))
$$

(see for example [2, Example 3.14.15]). Recall that a Banach space is said to have the UMD property or uniform martingale difference property if the Hilbert transform is bounded in $L^{p}(\mathbb{R}, X), 1<p<\infty$; see for example [2]. Every bounded cosine function in a UMD-space $X$ may be decomposed in this way (2, Section $3.16])$. 
At first glance, $C_{0}$-semigroups are connected with the first order Cauchy problem while cosine families are closer to the second order Cauchy problem; see more details in [2]. Now we introduce the following classes of cosine families.

Definition 0.1. A cosine function $\{C(t)\}_{t \geq 0}$ is hypercyclic if there exists $x \in$ $X$ such that $\{C(t) x\}_{t \geq 0}$ is dense in $X$; in this case the vector $x \in X$ is called hypercyclic. The cosine function $\{C(t)\}_{t \geq 0}$ is topologically transitive if for every pair of nonvoid sets $U, V$ there exists $t_{0}$ such that

$$
C\left(t_{0}\right) U \cap V \neq \emptyset \text {. }
$$

Also, $\{C(t)\}_{t \geq 0}$ is topologically mixing if for every pair of nonvoid sets $U, V \subset X$ there exists $t_{0}$ such that

$$
C(t) U \cap V \neq \emptyset \text { for all } t \text { such that } t \geq t_{0} .
$$

In the first section we give sufficient conditions for the hypercyclicity and topological mixing of a strongly continuous cosine function. We apply these results to study cosine functions defined by translation groups in the second section. We also prove that every separable infinite dimensional complex Banach space admits a topologically mixing uniformly continuous cosine function in the last section.

\section{TopologicAlLy TRANSITIVE AND MIXING CRITERIA}

The first result is a consequence of a general result of universality in the survey of Grosse-Erdmann [9, Theorem1]. The proof is given here for completeness.

Theorem 1.1. Let $\{C(t)\}_{t \geq 0}$ be a strongly continuous cosine function on a separable Banach space $X$. Then the following assertions are equivalent:

(i) $\{C(t)\}_{t \geq 0}$ is topologically transitive.

(ii) $\{C(t)\}_{t \geq 0}$ has a dense set of hypercyclic vectors.

Proof. We take the set of positive rational numbers and enumerate them as $\left\{t_{1}, t_{2}\right.$, $\ldots\}$. Consider now the sequence family $\left\{C\left(t_{n}\right)\right\}_{n \in \mathbb{N}}$. Take $p \in X$. Clearly the set $\{C(t) p\}_{t>0}$ is dense if and only if the set $\left\{C\left(t_{n}\right) p\right\}_{n \in \mathbb{N}}$ is dense.

Consider now the covering of $X$ by the enumerated sequence of balls $B_{m}$ centered on a countable subset of $X$ with rational radius. Also, define

$$
G_{m}:=\bigcup_{n \in \mathbb{N}} C\left(t_{n}\right)^{-1}\left(B_{m}\right) .
$$

Then the set of hypercyclic vectors for $\{C(t)\}_{t \geq 0}$ is equal to $\bigcap_{m \in \mathbb{N}} G_{m}$. This set is dense by the Baire category theorem if $\{C(t)\}_{t \geq 0}$ is topologically transitive and thus we show (i) $\Rightarrow$ (ii).

Conversely, if $\{C(t)\}_{t>0}$ has a dense set of hypercyclic vectors, each term of the intersection must be dense and thus $\{C(t)\}_{t \geq 0}$ is topologically transitive and we have proved (ii) $\Rightarrow$ (i).

In the next results, we give some topologically transitive criteria and one topologically mixing criterion for cosine functions. The following theorem can be shown from [6, Remark 2.6(3)] taking the particular case $T_{n}=C\left(t_{n}\right)$. However we also prefer to give the proof for completeness.

Theorem 1.2. Let $\{C(t)\}_{t \geq 0}$ be a strongly continuous cosine function on a separable Banach space $X$. If there exists a sequence of positive reals $\left\{t_{n}\right\}, t_{n} \rightarrow \infty$, such that 
(i) $X_{0}:=\left\{x \in X: \lim _{t_{n} \rightarrow \infty} C\left(t_{n}\right) x=0\right\}$ and

(ii) $X_{\infty}:=\left\{y \in X: \exists u_{n} \rightarrow 0, \lim _{t_{n} \rightarrow \infty} C\left(t_{n}\right) u_{n} \rightarrow y\right\}$

are dense, then $\{C(t)\}_{t \geq 0}$ is a topologically transitive cosine function.

Proof. Given $U$ and $V$ a pair of nonempty open subsets in $X$, since $X_{0}$ and $X_{\infty}$ are dense, there exist $x_{0} \in X_{0} \cap U$ and $y_{0} \in X_{\infty} \cap V$. One has $C\left(t_{n}\right) x_{0} \rightarrow 0$ and there exists $u_{n} \rightarrow 0$ with $\lim _{t_{n} \rightarrow \infty} C\left(t_{n}\right) u_{n} \rightarrow y_{0}$. Thus $C\left(t_{n}\right)\left(x_{0}+u_{n}\right) \rightarrow y_{0} \in V$. Since $U$ and $V$ are open, there exists $t^{\prime}>0$ such that $C\left(t^{\prime}\right) U \cap V \neq \emptyset$.

Corollary 1.3. Let $\{C(t)\}_{t \geq 0}$ be a strongly continuous cosine function on a separable Banach space $X$. If there exists a sequence of positive reals $\left\{t_{n}\right\}, t_{n} \rightarrow \infty$, such that $X_{1}:=\left\{x \in X: \lim _{t_{n} \rightarrow \infty} C\left(t_{n}\right) x=\lim _{t_{n} \rightarrow \infty} C\left(2 t_{n}\right) x=0\right\}$ is dense, then $\{C(t)\}_{t \geq 0}$ is a topologically transitive cosine function.

Proof. We consider the subspaces $X_{0}$ and $X_{\infty}$ defined in Theorem 1.2, Then $X_{1} \subset$ $X_{0}$ and $X_{1} \subset X_{\infty}$ because, given $y_{0} \in X_{1}$, we define $u_{n}=2 C\left(t_{n}\right) y_{0}$. Then $u_{n} \rightarrow 0$ and $\lim _{t_{n} \rightarrow \infty} C\left(t_{n}\right) u_{n}=\lim _{t_{n} \rightarrow \infty} y_{0}+C\left(2 t_{n}\right) y_{0} \rightarrow y_{0}$. Hence $X_{0}$ and $X_{\infty}$ are dense sets and the proof is obtained as a consequence of Theorem 1.2 .

Theorem 1.4. Let $\{C(t)\}_{t \geq 0}$ be a strongly continuous cosine function on a separable Banach space $X$. If $X_{0}:=\left\{x \in X: \lim _{t \rightarrow \infty} C(t) x=0\right\}$ is dense, then $\{C(t)\}_{t \geq 0}$ is a topologically mixing cosine function.

Proof. Given $U$ and $V$ a pair of nonempty open subsets in $X$, since $X_{0}$ is dense, there exist $x_{0} \in X_{0} \cap U$ and $y_{0} \in X_{0} \cap V$. One has $C(t) x_{0} \rightarrow 0$ as $t \rightarrow \infty$ and if we define $u_{t}:=2 C(t) y_{0}$, then $u_{t} \rightarrow 0$ and $C(t) u_{t}=y_{0}+C(2 t) y_{0}$. Thus $\lim _{t \rightarrow \infty} C(t) u_{t} \rightarrow y_{0}$. Thus $C(t)\left(x_{0}+u_{t}\right) \rightarrow y_{0} \in V$. Since $U$ and $V$ are open, there exists $t_{0}$ such that $C(t) U \cap V \neq \emptyset$, for all $t>t_{0}$.

\section{TOPOLOGICALlY TRANSITIVE AND MIXING TRANSLATIONS}

By an admissible weight function on $\mathbb{R}$ we mean a measurable function $\rho: \mathbb{R} \rightarrow \mathbb{R}$ satisfying the conditions

(i) $\rho(x)>0$ for all $x \in \mathbb{R}$;

(ii) there exist constants $M \geq 1$ and $\omega \in \mathbb{R}$ such that $\rho(x) \leq M e^{\omega t} \rho(t+x)$ for all $x \in \mathbb{R}$ and all $t>0$.

([8, Definition 4.1]). We consider the function spaces $\left(L_{\rho}^{p}(\mathbb{R}),\|\cdot\|_{p}\right)$,

$$
L_{\rho}^{p}(\mathbb{R})=\left\{u: \mathbb{R} \rightarrow \mathbb{C}: u \text { measurable, } \int_{\mathbb{R}}|u(\tau)|^{p} \rho(\tau) d \tau<\infty\right\},
$$

with

$$
\|u\|_{p}=\left\{\int_{\mathbb{R}}|u(\tau)|^{p} \rho(\tau) d \tau\right\}^{\frac{1}{p}}, \quad p \geq 1
$$

and $\left(C_{0, \rho}(\mathbb{R}),\|\cdot\|_{\infty}\right)$ where

$$
C_{0, \rho}(\mathbb{R})=\left\{u: \mathbb{R} \rightarrow \mathbb{C}: u \text { continuous, } \lim _{|\tau| \rightarrow \infty} \rho(\tau) u(\tau)=0\right\}
$$

with

$$
\|u\|_{\infty}=\sup _{\tau \in \mathbb{R}}|u(\tau)| \rho(\tau) .
$$


Lemma 2.1 ([8, Lemma 4.2]). Let $\rho$ be an admissible weight function on $\mathbb{R}$. For each $l>0$ there are constants $0<m \leq M$ (depending on $\rho$ and $l$ only) such that for each $\sigma \in \mathbb{R}$ and each $\tau \in[\sigma, \sigma+l]$

$$
m \rho(\sigma) \leq \rho(\tau) \leq M \rho(\sigma+l) .
$$

The translation semigroup $\{T(t)\}_{t \geq 0}$ with parameter $t \in[0, \infty)$ is defined as

$$
[T(t) u](\tau):=u(\tau+t) \text { for } u \in C_{0, \rho}(\mathbb{R}) \text { or } L_{\rho}^{p}(\mathbb{R}) .
$$

The $C_{0}$-semigroup $\{T(t)\}_{t \geq 0}$ was considered in [8, Section 4] and [3, Section 4].

Theorem 2.2. Let $X$ be one of the spaces $L_{\rho}^{p}(\mathbb{R})$ or $C_{0, \rho}(\mathbb{R})$ with an admissible weight function $\rho$. Moreover, let $\rho(-\tau)$ be an admissible weight function. Then the translation semigroup $\{T(t)\}_{t \geq 0}$ can be extended to a group on $X$ and

(i) $C(t)=\frac{1}{2}(T(t)+T(-t))$ is topologically mixing if and only if

$$
\lim _{|t| \rightarrow \infty} \rho(t)=0,
$$

(ii) the cosine function $C(t):=\frac{1}{2}(T(t)+T(-t))$ is topologically transitive if there exists a sequence $\left\{t_{j}\right\}_{j \geq 0}$ of positive real numbers such that

$$
\lim _{j \rightarrow \infty} \rho\left(t_{j}\right)=\lim _{j \rightarrow \infty} \rho\left(2 t_{j}\right)=\lim _{j \rightarrow \infty} \rho\left(-t_{j}\right)=\lim _{j \rightarrow \infty} \rho\left(-2 t_{j}\right)=0 .
$$

Proof. We will prove the case $X=L_{\rho}^{p}(\mathbb{R})$. First, we look at (i).

Suppose that $\lim _{|t| \rightarrow \infty} \rho(t)=0$. It is enough to prove that $\{C(t)\}_{t \geq 0}$ satisfies the condition of Theorem 1.4. Consider the set $X_{0}$ of all $C^{\infty}$-functions defined on $\mathbb{R}$ with compact support, which is a dense subspace of $X$. Let us see that $C(t) u$ converges to zero as $t \rightarrow \infty$ for all $u \in X_{0}$.

Let $u$ be a $C^{\infty}$-function whose support is contained in $[-l, l], l>0$, and let $t>2 l$. Following ideas similar to those in the proof of [8, Theorem 4.8], we have

$$
\begin{aligned}
\|C(t) u\|_{p}^{p} & =\int_{\mathbb{R}}\left|\frac{u(\tau+t)+u(\tau-t)}{2}\right|^{p} \rho(\tau) d \tau \\
& \leq M \max (\rho(-t), \rho(t)) \int_{-l}^{l}|u(\tau)|^{p} d \tau
\end{aligned}
$$

and

$$
\begin{aligned}
\|u\|_{p}^{p} & =\int_{-l}^{l}|u(\tau)|^{p} \rho(\tau) d \tau=\int_{-l}^{0}|u(\tau)|^{p} \rho(\tau) d \tau+\int_{0}^{l}|u(\tau)|^{p} \rho(\tau) d \tau \\
& \geq m_{1} \rho(0) \int_{0}^{l}|u(-\tau)|^{p} d \tau+m_{2} \rho(0) \int_{0}^{l}|u(\tau)|^{p} d \tau \\
& \geq \min \left(m_{1}, m_{2}\right) \rho(0) \int_{-l}^{l}|u(\tau)|^{p} d \tau .
\end{aligned}
$$

Hence

$$
\|C(t) u\|_{p}^{p} \leq M \frac{\max (\rho(-t), \rho(t))}{\min \left(m_{1}, m_{2}\right) \rho(0)}\|u\|_{p}^{p} .
$$

Since $\lim _{|t| \rightarrow \infty} \rho(t)=0$, we have that $\|C(t) u\|_{p} \rightarrow 0$ as $t \rightarrow \infty$.

Suppose now that $C(t)$ is topologically mixing. Then given $l>0$ and $f \in X$ with supp $f \subset[-l, l], f \geq 0$, and $\|f\|_{p}=1$, we have $\forall \varepsilon>0, \exists t_{\varepsilon}>2 l$ such that $\forall t>t_{\varepsilon} \exists v_{t} \in X$,

$$
\left\|v_{t}-f\right\|_{p}<\varepsilon \text { and }\left\|C(t) v_{t}\right\|_{p}<\varepsilon .
$$


We define

$$
\begin{gathered}
v_{t}^{+}:=\max \left(0, v_{t}\right) \geq 0, \\
w_{t}:=v_{t}^{+} \chi_{[-l, l]} .
\end{gathered}
$$

We obtain that $\left\|C(t) w_{t}\right\|_{p}<\varepsilon$ and $\left\|w_{t}\right\|_{p}>1-\varepsilon$. Moreover

$$
\begin{aligned}
\left\|C(t) w_{t}\right\|_{p}^{p} & =\left(\frac{1}{2}\right)^{p}\left(\int_{-l-t}^{l-t}\left(w_{t}(s+t)\right)^{p} \rho(s) d s+\int_{-(l+t)}^{l-t}\left(w_{t}(-s-t)\right)^{p} \rho(-s) d s\right) \\
& \geq\left(m_{2} \rho(-l-t)+m_{1} \rho(-(-(l+t)))\right) \int_{-l}^{l}\left|w_{t}(s)\right|^{p} d s \\
& \left.\geq\left(m_{2} m_{1} \rho(-t)+m_{1} m_{2} \rho(t)\right) \int_{-l}^{l}\left|w_{t}(s)\right|^{p} d s\right)
\end{aligned}
$$

and

$$
\begin{aligned}
\left\|w_{t}\right\|_{p}^{p} & =\int_{0}^{l}\left(w_{t}(-s)\right)^{p} \rho(-s) d s+\int_{0}^{l}\left(w_{t}(s)\right)^{p} \rho(s) d s \\
& \leq\left(M_{1} \rho(-l)+M_{2} \rho(l)\right) \int_{-l}^{l}\left|w_{t}(s)\right|^{p} d s ;
\end{aligned}
$$

see similar ideas in [8, Theorem 4.8]. Hence

$$
\varepsilon^{p}>\left\|C(t) w_{t}\right\|_{p}^{p} \geq \frac{m_{1}^{2} \rho(-t)+m_{2}^{2} \rho(t)}{M_{1} \rho(-l)+M_{2} \rho(l)}\left\|w_{t}\right\|_{p}^{p}>\frac{m_{1} m_{2}(\rho(-t)+\rho(t))}{M_{1} \rho(-l)+M_{2} \rho(l)}(1-\varepsilon)^{p} .
$$

Thus, we have

$$
\lim _{|t| \rightarrow \infty} \rho(t)=0 .
$$

In order to prove (ii), we consider $X_{1}$ as the set of all $C^{\infty}$-functions defined on $\mathbb{R}$ with compact support, which is a dense subspace of $X$. By Corollary 1.3 , we obtain (ii).

\section{ON The EXISTENCE OF TOPOLOGICALLY MIXING COSINE FUNCTIONS}

We consider the weighted $\ell^{1}$-space with a weight sequence $\beta=\left(\beta_{i}\right)_{i=1}^{\infty}$ of positive numbers defined by

$$
\ell^{1}(\beta):=\left\{\left(x_{i}\right)_{i=1}^{\infty}: x_{i} \in \mathbb{C}, \sum_{i=1}^{\infty} \beta_{i}\left|x_{i}\right|<\infty\right\}
$$

equipped with the norm

$$
\left\|\left(x_{i}\right)\right\|:=\sum_{i=1}^{\infty} \beta_{i}\left|x_{i}\right| .
$$

If $\sup _{i \in \mathbb{N}} \frac{\beta_{i}}{\beta_{i+1}} \leq M$ for some constant $M$, then the backward shift $B$ defined by

$$
B\left(x_{1}, x_{2}, \cdots\right):=\left(x_{2}, x_{3}, \cdots\right)
$$

is a bounded linear operator on $\ell^{1}(\beta)$.

Following some ideas of [3, Lemma 2.1], where it was proved that $\left\{e^{t B^{2}}\right\}_{t \geq 0}$ is topologically mixing in $\ell^{1}(\beta)$, we will prove that $\left\{C(t)=\frac{1}{2}\left(e^{t B}+e^{-t B}\right)\right\}_{t \geq 0}$ is also a topologically mixing cosine function. 
Lemma 3.1. Let $\beta=\left(\beta_{i}\right)_{i=1}^{\infty}$ be a sequence of positive numbers and let $B$ be the backward shift operator. If $\sup _{i \in \mathbb{N}} \frac{\beta_{i}}{\beta_{i+1}} \leq M$ for some constant $M$, then the uniformly continuous cosine function $\left\{C(t)=\frac{1}{2}\left(e^{t B}+e^{-t B}\right)\right\}_{t \geq 0}$ is topologically mixing in $\ell^{1}(\beta)$.

Proof. Consider

$$
\varphi:=\left\{\left(x_{i}\right)_{i=1}^{\infty} \subset \mathbb{C}: \exists m, \quad x_{i}=0 \quad \forall i>m\right\},
$$

which is dense in $\ell^{1}(\beta)$. Given any pair $y=\left(y_{i}\right)_{i=1}^{\infty}$ and $z=\left(z_{i}\right)_{i=1}^{\infty}$ of elements in $\varphi$, and $\varepsilon>0$, our purpose is to construct vectors $v(t)=\left(v_{i}(t)\right)_{i=1}^{\infty} \in \ell^{1}(\beta), t \geq 0$, such that there exists $t_{0} \geq 0$ with

$$
\|v(t)-y\|<\varepsilon \text { and }\|C(t) v(t)-z\|<\varepsilon \text {, for all } t>t_{0} .
$$

To do this, we first select and fix $k \in \mathbb{N}$ with

$$
y_{i}=z_{i}=0 \text { for } i \geq 2 k .
$$

We now define $v(t)$ as follows:

$$
v_{i}(t)= \begin{cases}y_{i} & \text { for } i=1, \ldots, 2 k, \\ 0 & \text { for } i=2 k+1, \ldots, 4 k \text { and for } i>6 k .\end{cases}
$$

In order to define $v_{i}(t)$ for $i=4 k+1, \ldots, 6 k$, we use that

$$
(C(t) v(t))_{i}=\sum_{j=0}^{3 k} \frac{t^{2 j}}{(2 j) !} v_{2 j+i}(t) \text { for } i=1, \ldots, 2 k .
$$

In fact, we choose $v_{i}(t)$ for $i=4 k+1, \ldots, 6 k$ as the solutions of the following system:

$$
A\left(\begin{array}{c}
y_{1} \\
\vdots \\
y_{2 k}
\end{array}\right)+D\left(\begin{array}{c}
v_{4 k+1}(t) \\
\vdots \\
v_{6 k}(t)
\end{array}\right)=\left(\begin{array}{c}
z_{1} \\
\vdots \\
z_{2 k}
\end{array}\right)
$$

where

$$
A:=\left(\begin{array}{cccccccccc}
1 & 0 & \mu_{1} & 0 & \mu_{2} & 0 & \cdots & 0 & \mu_{k-1} & 0 \\
0 & 1 & 0 & \mu_{1} & 0 & \mu_{2} & \cdots & \mu_{k-2} & 0 & \mu_{k-1} \\
\cdot & 0 & 1 & 0 & \mu_{1} & 0 & \cdots & 0 & \mu_{k-2} & 0 \\
. & . & 0 & 1 & 0 & \mu_{1} & \cdots & . & 0 & \mu_{k-2} \\
. & . & . & 0 & 1 & 0 & \cdots & . & . & 0 \\
. & . & . & . & 0 & 1 & \cdots & . & . & . \\
\vdots & \vdots & \vdots & \vdots & \vdots & \vdots & & \vdots & \vdots & \vdots \\
0 & 0 & 0 & 0 & 0 & 0 & \cdots & 1 & 0 & \mu_{1} \\
0 & 0 & 0 & 0 & 0 & 0 & \cdots & 0 & 1 & 0 \\
0 & 0 & 0 & 0 & 0 & 0 & \cdots & 0 & 0 & 1
\end{array}\right)
$$


with $\mu_{i}=\frac{t^{2 i}}{(2 i) !}$ for $i=1, \ldots, k-1$ and

$$
D:=\left(\begin{array}{ccccccc}
\lambda_{0} & 0 & \lambda_{1} & 0 & \cdots & \lambda_{k-1} & 0 \\
0 & \lambda_{0} & 0 & \lambda_{1} & \cdots & 0 & \lambda_{k-1} \\
\lambda_{-1} & 0 & \lambda_{0} & 0 & \cdots & . & 0 \\
0 & \lambda_{-1} & 0 & \lambda_{0} & \cdots & . & . \\
\vdots & \vdots & \vdots & \vdots & & \vdots & \vdots \\
\lambda_{-k+1} & 0 & . & . & \cdots & \lambda_{0} & 0 \\
0 & \lambda_{-k+1} & . & . & \cdots & 0 & \lambda_{0}
\end{array}\right)
$$

where $\lambda_{i}=\frac{t^{4 k+2 i}}{(4 k+2 i) !}$ for $i=-k+1, \ldots, k-1$.

Observe that $D=U W S$ where

$$
\begin{aligned}
U & =\left(\begin{array}{ccccccc}
t^{4 k} & 0 & 0 & 0 & \cdots & 0 & 0 \\
0 & t^{4 k} & 0 & 0 & \cdots & 0 & 0 \\
0 & 0 & t^{4 k-2} & 0 & \cdots & 0 & 0 \\
0 & 0 & 0 & t^{4 k-2} & \cdots & 0 & 0 \\
\vdots & \vdots & \vdots & \vdots & & \vdots & \vdots \\
0 & 0 & 0 & 0 & \cdots & t^{2 k+2} & 0 \\
0 & 0 & 0 & 0 & \cdots & 0 & t^{2 k+2}
\end{array}\right), \\
W & :=\left(\begin{array}{ccccccc}
\eta_{0} & 0 & \eta_{1} & 0 & \cdots & \eta_{k-1} & 0 \\
0 & \eta_{0} & 0 & \eta_{1} & \cdots & 0 & \eta_{k-1} \\
\eta_{-1} & 0 & \eta_{0} & 0 & \cdots & . & 0 \\
0 & \eta_{-1} & 0 & \eta_{0} & \cdots & . & . \\
\vdots & \vdots & \vdots & \vdots & & \vdots & \vdots \\
\eta_{-k+1} & 0 & . & . & \cdots & \eta_{0} & 0 \\
0 & \eta_{-k+1} & . & . & \cdots & 0 & \eta_{0}
\end{array}\right)
\end{aligned}
$$

with $\eta_{i}=\frac{1}{(4 k+2 i) !}$ for $i=-k+1, \ldots, k-1$ and

$$
S=\left(\begin{array}{ccccccc}
1 & 0 & 0 & 0 & \cdots & 0 & 0 \\
0 & 1 & 0 & 0 & \cdots & 0 & 0 \\
0 & 0 & t^{2} & 0 & \cdots & 0 & 0 \\
0 & 0 & 0 & t^{2} & \cdots & 0 & 0 \\
\vdots & \vdots & \vdots & \vdots & & \vdots & \vdots \\
0 & 0 & 0 & 0 & \cdots & t^{2 k-2} & 0 \\
0 & 0 & 0 & 0 & \cdots & 0 & t^{2 k-2}
\end{array}\right)
$$

are regular matrices with $2 k$ rows and $2 k$ columns for $t \neq 0$. Thus the solution of system (3.1) is given by

$$
\left(\begin{array}{c}
v_{4 k+1}(t) \\
\vdots \\
v_{6 k}(t)
\end{array}\right)=S^{-1} W^{-1} U^{-1}\left[\left(\begin{array}{c}
z_{1} \\
\vdots \\
z_{2 k}
\end{array}\right)-A\left(\begin{array}{c}
y_{1} \\
\vdots \\
y_{2 k}
\end{array}\right)\right] .
$$

Let $\left(w_{i}\right)_{i=1}^{2 k}$ be defined as

$$
\left(\begin{array}{c}
w_{1} \\
\vdots \\
w_{2 k}
\end{array}\right)=U^{-1}\left[\left(\begin{array}{c}
z_{1} \\
\vdots \\
z_{2 k}
\end{array}\right)-A\left(\begin{array}{c}
y_{1} \\
\vdots \\
y_{2 k}
\end{array}\right)\right] .
$$


Then, if we consider $t$ with $t>1$, there exists $C_{1}$ independent of $t$ such that

$$
\left|w_{j}\right| \leq C_{1}|t|^{-2 k-2} \text { for } j=1, \ldots, 2 k .
$$

Thus there exists $C_{2}$ independent of $t$ satisfying

$$
\left|v_{i}(t)\right| \leq C_{2}|t|^{2 k-i} \text { for } i=4 k+1, \ldots, 6 k .
$$

Since $v_{i}(t)=y_{i}$ for $i \leq 4 k$ and $y_{i}=0$ for $i>2 k$, we have

$$
\|v(t)-y\|=\sum_{i=4 k+1}^{6 k} \beta_{i}\left|v_{i}(t)\right| \leq C_{2} \sum_{i=4 k+1}^{6 k} \beta_{i}|t|^{-2 k} .
$$

Hence, for sufficiently large $|t|$, we get

$$
\|v(t)-y\|<\varepsilon .
$$

Moreover

$$
\begin{gathered}
\|C(t) v(t)-z\|=\sum_{i=2 k+1}^{6 k} \beta_{i}\left|(C(t) v(t))_{i}\right|=\sum_{i=2 k+1}^{6 k} \beta_{i}\left|\sum_{j=0}^{2 k-1} \frac{t^{2 j}}{(2 j) !} v_{2 j+i}(t)\right| \\
\leq \sum_{i=2 k+1}^{6 k} \beta_{i}\left(\sum_{j=0}^{2 k-1} \frac{|t|^{2 j}}{(2 j) !}|t|^{2 k-2 j-i}\right) \leq \sum_{i=2 k+1}^{6 k} \beta_{i}\left(\sum_{j=0}^{2 k-1} \frac{|t|^{-1}}{(2 j) !}\right) .
\end{gathered}
$$

Therefore we conclude

$$
\|C(t) v(t)-z\|<\varepsilon
$$

if $|t|$ is sufficiently large.

The proof of the following lemma is similar to [10, Lemma 2.1].

Lemma 3.2. Let $X_{1}, X_{2}$ be separable Banach spaces, let $\Phi: X_{1} \rightarrow X_{2}$ be a continuous mapping with dense range, and let $\{C(t)\}_{t \geq 0}$ and $\{\tilde{C}(t)\}_{t \geq 0}$ be strongly continuous cosine functions on $X_{1}$ and $X_{2}$, respectively, such that $\tilde{C}(t) \Phi=\Phi C(t)$ for all $t \geq 0$. If $\{C(t)\}_{t \geq 0}$ is topologically mixing (topologically transitive, hypercyclic), then $\{\tilde{C}(t)\}_{t \geq 0}$ is also topologically mixing (topologically transitive, hypercyclic).

The following result due to Ovsepian and Pełcyński [11 is needed to show the existence result.

Theorem 3.3 ([11]). Let $X$ be a separable infinite dimensional Banach space. Then there exist $\left(x_{n}\right)_{n=1}^{\infty} \subset X$ and $\left(f_{m}\right)_{m=1}^{\infty} \subset X^{*}$, the dual space of $X$, satisfying the following conditions:

(1) $f_{m}\left(x_{n}\right)=\delta_{m, n}, m, n \in \mathbb{N}$.

(2) $\overline{\operatorname{span}\left\{x_{n}: n \in \mathbb{N}\right\}}=X$.

(3) $f_{m}(x)=0$, for all $m \in \mathbb{N} \Rightarrow x=0$.

(4) $\left\|x_{n}\right\|=1$, for all $n \in \mathbb{N}$ and $\sup _{m \in \mathbb{N}}\left\|f_{m}\right\|=c<\infty$.

Theorem 3.4. Every separable infinite dimensional Banach space $X$ admits a topologically mixing uniformly continuous cosine function.

Proof. Let $\left(x_{n}\right)_{n=1}^{\infty} \subset X$ and $\left(f_{m}\right)_{m=1}^{\infty} \subset X^{*}$ as in Theorem 3.3. Consider the bounded linear operator $S: X \rightarrow X$ defined by

$$
S x:=\sum_{n=1}^{\infty} \frac{1}{2^{n}} f_{n+1}(x) x_{n} .
$$


Our purpose is to show that $\left\{\frac{1}{2}\left(e^{t S}+e^{-t S}\right)\right\}_{t \geq 0}$ is a topologically mixing uniformly continuous cosine function.

Define $\Phi: \ell^{1} \rightarrow X$, by $\Phi\left(\left(\alpha_{j}\right)_{j}\right):=\sum_{j=1}^{\infty} \alpha_{j} x_{j}$, which is a bounded linear operator with dense range.

Claim. The cosine function $\frac{1}{2}\left(e^{t \tilde{S}}+e^{-t \tilde{S}}\right): \ell^{1} \rightarrow \ell^{1}$ is topologically mixing, where

$$
\tilde{S}\left(\left(\alpha_{j}\right)_{j}\right):=\left(\frac{\alpha_{2}}{2}, \cdots, \frac{\alpha_{n+1}}{2^{n}}, \cdots\right) .
$$

From the definition it follows that $S \Phi=\Phi \tilde{S}$ on $\ell^{1}$ and therefore $\frac{1}{2}\left(e^{t S}+e^{-t S}\right) \Phi=$ $\Phi \frac{1}{2}\left(e^{t \tilde{S}}+e^{-t \tilde{S}}\right)$ for all $t \geq 0$. Applying Lemma 3.2, we have that $\left\{\frac{1}{2}\left(e^{t S}+e^{-t S}\right)\right\}_{t \geq 0}$ is topologically mixing in $X$.

The proof of the claim is similar to the proof of [3, Theorem 2.4] where we use Lemma 3.1 and Lemma 3.2 .

\section{REFERENCES}

[1] S. I. Ansari, Existence of hypercyclic operators on topological vector spaces, J. Funct. Anal. 148 (1997), no. 2, 384-390. MR1469346 (98h:47028a)

[2] W. Arendt, C. Batty, M. Hieber, and F. Neubrander, Vector-valued Laplace Transforms and Cauchy problems, Birkhäuser, 2001. MR1886588(2003g:47072)

[3] T. Bermúdez, A. Bonilla, J. A. Conejero, and A. Peris, Hypercyclic, topologically mixing and chaotic semigroups in Banach spaces, Studia Math. 170 (2005), no. 1, 57-75. MR2142183 (2005m:47013)

[4] T. Bermúdez, A. Bonilla, and A. Martinón, On the existence of chaotic and hypercyclic semigroups in Banach spaces, Proc. Amer. Math. Soc. 131 (2003), no. 8, 2435-2441. MR.1974641 (2004b:47006)

[5] L. Bernal-González, On hypercyclic operators on Banach spaces, Proc. Amer. Math. Soc. 127 (1999), no. 4, 1003-1010. MR.1476119 (99f:47010)

[6] J. Bès and A. Peris, Hereditarily hypercyclic operators, J. Funct. Anal. 167 (1999), no. 1, 94-112. MR 1710637 (2000f:47012)

[7] J. Bonet and A. Peris, Hypercyclic operators on non-normable Fréchet spaces, J. Funct. Anal. 159 (1998), no. 2, 587-595. MR1658096 (99k:47044)

[8] W. Desch, W. Schappacher, and G. F. Webb, Hypercyclic and chaotic semigroups of linear operators, Ergodic Theory and Dynamical Syst. 17 (1997), 1-27. MR.1468101 (98j:47083)

[9] K. G. Grosse-Erdmann, Universal families and hypercyclic operators, Bull. Amer. Math. Soc. 36(1999), no. 3, 345-381. MR1685272 (2000c:47001)

[10] F. Martínez-Giménez and A. Peris, Chaos for backward shift operators, Internat. J. Bifur. Chaos Appl. Sci. Engrg. 12 (2002), no. 8, 1703-1715. MR1927407 (2003h:47056)

[11] R. I. Ovsepian and A. Pełczyński, On the existence of a fundamental total and bounded biorthogonal sequence in every separable Banach space, and related constructions of uniformly bounded orthonormal systems in $l^{2}$, Studia Math. 54 (1975), 149-159. MR0394137 $(52: 14942)$

[12] S. Rolewicz, On orbits of elements, Studia Math. 32 (1969), 17-22. MR0241956 (39:3292)

Departamento de Análisis Matemático, Universidad de La Laguna, 38271 La Laguna (TENERIFE), Spain

E-mail address: abonilla@ull.es

Departamento de Matemáticas, Universidad de Zaragoza, 50009 Zaragoza, Spain

E-mail address: pjmiana@unizar.es 